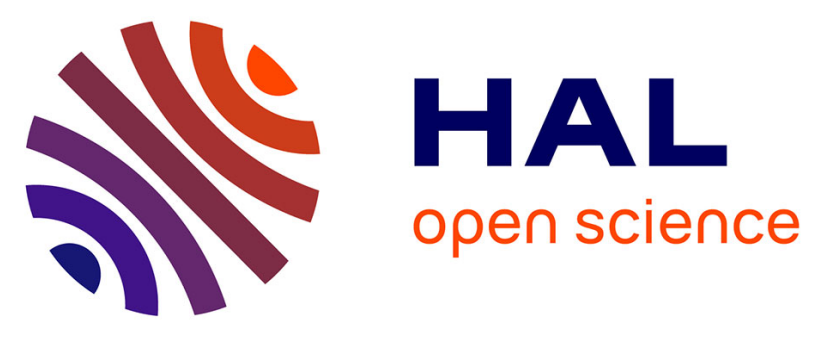

\title{
Relation between baseline LDL-cholesterol and cardiovascular outcomes in high cardiovascular risk hypertensive patients: A post-hoc SPRINT data analysis
}

Lee Nguyen, Niki Procopi, Joe-Elie Salem, Pierre Squara, Christian Funck-Brentano

\section{To cite this version:}

Lee Nguyen, Niki Procopi, Joe-Elie Salem, Pierre Squara, Christian Funck-Brentano. Relation between baseline LDL-cholesterol and cardiovascular outcomes in high cardiovascular risk hypertensive patients: A post-hoc SPRINT data analysis. International Journal of Cardiology, 2019, 286, pp.159161. 10.1016/j.ijcard.2019.01.048 . hal-02136104

\section{HAL Id: hal-02136104 \\ https://hal.sorbonne-universite.fr/hal-02136104}

Submitted on 3 Jul 2019

HAL is a multi-disciplinary open access archive for the deposit and dissemination of scientific research documents, whether they are published or not. The documents may come from teaching and research institutions in France or abroad, or from public or private research centers.
L'archive ouverte pluridisciplinaire HAL, est destinée au dépôt et à la diffusion de documents scientifiques de niveau recherche, publiés ou non, émanant des établissements d'enseignement et de recherche français ou étrangers, des laboratoires publics ou privés. 


\section{Relation between baseline LDL-cholesterol and cardiovascular}

\section{outcomes in high cardiovascular risk hypertensive patients: a}

\section{post-hoc SPRINT data analysis}

Lee S. Nguyen ${ }^{1,2}$, MD,MSc ; Niki Procopi ${ }^{1}$, MD; Joe-Elie Salem², MD,PhD ;

Pierre Squara ${ }^{1}, \mathrm{MD}$ and Christian Funck-Brentano ${ }^{2 *}, \mathrm{MD}, \mathrm{PhD}$.

${ }^{1}$ CMC Ambroise Paré, Critical Care Medicine Department, Neuilly-sur-Seine, France

This author takes responsibility for all aspects of the reliability and freedom from

bias of the data presented and their discussed interpretation

2 Sorbonne Université, INSERM CIC Paris-Est, AP-HP, ICAN, Pitié-Salpêtrière Hospital,

Department of Pharmacology, F-75013 Paris, France

This author takes responsibility for all aspects of the reliability and freedom from

bias of the data presented and their discussed interpretation

*Corresponding author:

C. Funck-Brentano: Department of Pharmacology, Pitié-Salpêtrière University

Hospital, 75013 - Paris, France,

Telephone: +331421616 61; fax: +331421616 88;

e-mail: christian.funck-brentano@aphp.fr

Word count: 1042

Declarations of interest: none. 


\section{Abstract (250 words)}

Background. Patients at increased cardiovascular (CV) risk, noticeably hypertensive patients, have multiple CV risk factors which may be treatment targets. LDLcholesterol is one of such targets. Using the SPRINT cohort, studying the cardiovascular outcomes of hypertensive patients at increased CV risk, this post-hoc study aimed to assess the association of LDL-C with CV outcomes.

Methods. Clinical outcomes were those defined in SPRINT: a composite of various CV outcomes, all-cause mortality, and CV mortality. Association between LDL-C and the primary outcome was analysed using survival regression adjusted on confounding factors (age, sex, body-mass index, active smoking status, eGFRestimated kidney function, history of CV disease, Framingham risk score, SPRINT treatment arm (intensive or control), baseline high-density-lipoprotein-bound cholesterol, and co-treatments by aspirin and statins).

Results. LDL-C was not associated with the primary outcome in the overall cohort ( $n=9631)$. Among patients in secondary prevention (i.e. with a previous history of CV disease) ( $n=1562)$, LDL-C was marginally associated with the incidence of the primary outcome (adjusted hazard-ratio 1.005 (95\% Cl=1.002-1.009), p=0.005 (per $1 \mathrm{mg} / \mathrm{dl}$ increase)) however, discrimination was poor with a ROC AUC of $0.54, p=0.087$. There was no association between LDL-C and the primary outcome in other subgroup analyses (those under statin or not, and those in primary prevention).

Conclusion. This post-hoc analysis of SPRINT indicates that LDL-C levels do not influence cardiovascular events over a period of 3 years in a large cohort of 
hypertensive patients at increased risk of cardiovascular events but without previous history of clinical cardiovascular disease other than stroke.

Key words: Cardiovascular Diseases/prevention \& control; Hypertension/drug therapy; Dyslipidemias; Cholesterol, LDL; Hypolipidemic Agents; Treatment Outcome 


\section{Introduction}

Cardiovascular (CV) disease prevention relies in part on decreasing circulating low-density lipoprotein-bound cholesterol (LDL-C). Treatments include statins, antiPCSK9 antibodies, ezetimibe. Although indications for these treatments are welldefined, no consensus was achieved regarding a target LDL-C level.[1]

This study uses data from the Systolic Blood Pressure Intervention Trial (SPRINT) to assess CV risk associated with target and non-target LDL-C levels at the time of inclusion.[2]

\section{Methods}

In the SPRINT trial, 9361 hypertensive patients were randomly allocated to intensive or standard antihypertensive treatment strategies. Clinical outcomes were monitored for up to 6 years after inclusion and included a primary composite endpoint (including myocardial infarction (MI), acute coronary syndrome (ACS) not resulting in $\mathrm{MI}$, stroke, acute decompensated heart failure, and/or death from cardiovascular causes), all-cause mortality and cardiovascular mortality. All patients were at increased risk of CV events defined by one or more of the following: age $>75$ years, history of CV disease other than stroke (patients with prior stroke were excluded), chronic kidney disease with estimated glomerular filtration rate (eGFR) between 20 and less than $60 \mathrm{ml} / \mathrm{min} / 1.73 \mathrm{~m}^{2}$ or 10 -year Framingham risk score $>15 \%$.

Using the same dataset, this study focuses on post-hoc analysis of the association between LDL-C and the incidence of the primary composite endpoint of 
SPRINT. Data were obtained after qualifying for the worldwide data-analysis challenge organized by the New England Journal of Medicine.[3]

Data are presented as mean \pm standard deviation. Cox proportional-hazards regressions were performed to analyze time-to-endpoints. Confounding variables that were used for adjustment in multivariable analyses included age, sex, bodymass index, active smoking status, eGFR-estimated kidney function, history of CV disease, Framingham risk score, SPRINT treatment arm (intensive or control), baseline high-density-lipoprotein-bound cholesterol (HDL-C), and co-treatments with aspirin and/or statins. Discriminative performance of LDL-C regarding the prognostication of events (primary endpoint, all-cause mortality and CV mortality), i.e. the capacity of LDL to differentiate patients who develop the outcome from those who would not, was assessed by area under curve (AUC) of receiver-operator characteristics (ROC) curves. All analyses were performed using SPSS v23.0 (IBM, Armonk, USA).

\section{Results}

Patients' baseline characteristics are presented in the original article.[2] LDLC at baseline was $112.1 \pm 35.8 \mathrm{mg} / \mathrm{dl}$. Patients under statin medication represented 43.3\% of the cohort ( $n=4054)$. Patients who had a prior history of CV disease represented $16.7 \%$ of the cohort $(n=1562)$. There were $38(0.4 \%)$ missing baseline values for LDL-C.

In the overall SPRINT cohort ( $n=9361)$, multivariable Cox regression analyses showed no independent association between baseline LDL-C and the primary 
outcome. No threshold of LDL-C was associated with a higher risk of CV events

(Figure 1). Receiver operating characteristic (ROC) analysis of LDL-C found an area under curve of 0.47 for the incidence of the primary outcome. Furthermore, analyses on separate clinical events included in the primary endpoint (i.e. coronary events, stroke, CV mortality and all-cause mortality) did not reveal independent association between LDL-C and these events. There was no interaction between LDL-C and SPRINT treatment arm.

In a multivariable subgroup analysis limited to patients with a previous history of clinical CV disease other than stroke at the time of inclusion ( $n=1562$, with 7 missing values), there was a statistically significant association between LDL-C and the primary outcome with an adjusted hazard ratio of $1.005(95 \mathrm{Cl}=1.002-1.009)$, $\mathrm{p}=0.005$ (per $1 \mathrm{mg} / \mathrm{dl}$ increase). Baseline LDL-C was lower in this subgroup than in the overall cohort $(94.6 \pm 35.4$ vs. $112.1 \pm 35.8, p<0.00001)$. However, ROC analysis did not reach significance with an AUC of $0.54(p=0.087)$. The corresponding survival curves are shown in Figure 2.

Other subgroup analyses did not reveal associations between LDL-C and the primary outcome. Specifically, patients under statin therapy $(n=4054)$ did not show any association between LDL-C and clinical events and corresponding ROC AUC was $0.47(p=0.10)$

\section{Discussion}

There are several limitations to these results: i) SPRINT was a trial in hypertension and did not intend to test the hypothesis that LDL-C influences clinical 
outcomes; ii) a median follow-up duration of 3.26 years may have been too short to allow for a sufficient number of clinical events (i.e. the primary composite endpoint, or its components) to occur and sufficient statistical power to detect a significant association between LDL-C and outcomes, although LDL-C lowering strategies showed positive outcomes even during the first year of implementation,[4] and recent meta-analysis pooling 34 trials and 270,288 patients showed a positive effect with same median follow-up to that of SPRINT (3.9 years).[5] Moreover, ROC analyses were also in favor of a lack of LDL-C threshold more at risk than another, iii) there was no information on statin prescription changes nor duration under statin treatment after inclusion in SPRINT, and v) as with all multivariable analyses, adjustments were only made on variables available at baseline and other confounding variables may have been ignored.

Nevertheless, the most important finding of this analysis is that no LDL-C threshold was associated more than another, with CV events. In the whole cohort, patients with LDL-C $\geq 160 \mathrm{mg} / \mathrm{dl}$ at the beginning of the study did not experience more CV events than those with LDL-C $<70 \mathrm{mg} / \mathrm{dl}$. This association between LDL-C and CV events, however, existed in patients who had a prior history of CV disease. This supports contemporary recommendations which emphasize secondary prevention with aggressive LDL-C lowering irrespective of baseline levels. [6-8] This is even more relevant since the amplitude of risk reduction is proportional to the absolute LDL-C reduction, while LDL-C reduction is proportional to baseline LDL-C.[5] In the recent Standard vs. Intensive Statin Therapy for Hypercholesterolemic Patients with Diabetic Retinopathy (EMPATHY) randomized trial comparing intensive vs. standard statin therapy, more than half of patients in the intensive group failed 
to reach the target value of $70 \mathrm{mg} / \mathrm{dl}$ despite 3 years of treatment.[9] While this may partly explain why there was no statistical significance between the two treatment arms $(p=0.15)$, it also emphasizes the impracticability of such a target approach. This comes in addition to previous analyses from real-world databases,[10] and registry highlighting the difficulty of reaching pre-specified targets.[11]

\section{Conclusion}

In conclusion, this post-hoc analysis of SPRINT indicates that LDL-C levels do not influence cardiovascular events over a period of 3 years in a large cohort of hypertensive patients at increased risk of cardiovascular events but without previous history of clinical cardiovascular disease other than stroke. 


\section{REFERENCES}

[1] Waters DD, Boekholdt SM. An Evidence-Based Guide to Cholesterol-Lowering Guidelines. Can J Cardiol. 2017;33:343-9.

[2] Wright JT, Jr., Williamson JD, Whelton PK, et al. A Randomized Trial of Intensive versus Standard Blood-Pressure Control. N Engl J Med. 2015;373:2103-16.

[3] Burns NS, Miller PW. Learning What We Didn't Know - The SPRINT Data Analysis Challenge. N Engl J Med. 2017;376:2205-7.

[4] Baigent C, Blackwell L, Emberson J, et al. Efficacy and safety of more intensive lowering of LDL cholesterol: a meta-analysis of data from 170,000 participants in 26 randomised trials. Lancet. 2010;376:1670-81.

[5] Navarese EP, Robinson JG, Kowalewski M, et al. Association Between Baseline LDL-C Level and Total and Cardiovascular Mortality After LDL-C Lowering: A Systematic Review and Meta-analysis. Jama. 2018;319:1566-79.

[6] Catapano AL, Graham I, De Backer G, et al. 2016 ESC/EAS Guidelines for the Management of Dyslipidaemias. Eur Heart J. 2016;37:2999-3058.

[7] Murphy SA, Cannon CP, Wiviott SD, McCabe CH, Braunwald E. Reduction in recurrent cardiovascular events with intensive lipid-lowering statin therapy compared with moderate lipid-lowering statin therapy after acute coronary syndromes from the PROVE IT-TIMI 22 (Pravastatin or Atorvastatin Evaluation and Infection Therapy-Thrombolysis In Myocardial Infarction 22) trial. J Am Coll Cardiol. 2009;54:2358-62.

[8] Long-Term Intervention with Pravastatin in Ischaemic Disease (LIPID) Study Group. Prevention of cardiovascular events and death with pravastatin in patients with coronary heart disease and a broad range of initial cholesterol levels. N Engl J Med. 1998;339:1349-57.

[9] Itoh H, Komuro I, Takeuchi M, Akasaka T, et al. Intensive Treat-to-Target Statin Therapy in High-Risk Japanese Patients With Hypercholesterolemia and Diabetic Retinopathy: Report of a Randomized Study. Diabetes Care. 2018;41:1275-84.

[10] Krobot KJ, Yin DD, Alemao E, Steinhagen-Thiessen E. Real-world effectiveness of lipid-lowering therapy in male and female outpatients with coronary heart disease: relation to pre-treatment low-density lipoprotein-cholesterol, pretreatment coronary heart disease risk, and other factors. Eur J Cardiovasc Prev Rehabil. 2005;12:37-45.

[11] Yan AT, Yan RT, Tan M, et al. Contemporary management of dyslipidemia in high-risk patients: targets still not met. Am J Med. 2006;119:676-83. 
Figure 1. Cumulative survival curves regarding primary outcome in the overall

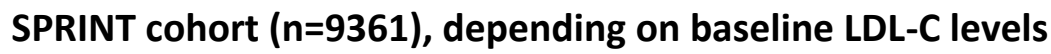

There was no association between baseline LDL-C levels and primary outcome, after adjusting for confounding factors (see text). There were $38(0.4 \%)$ baseline missing values for LDL-C.

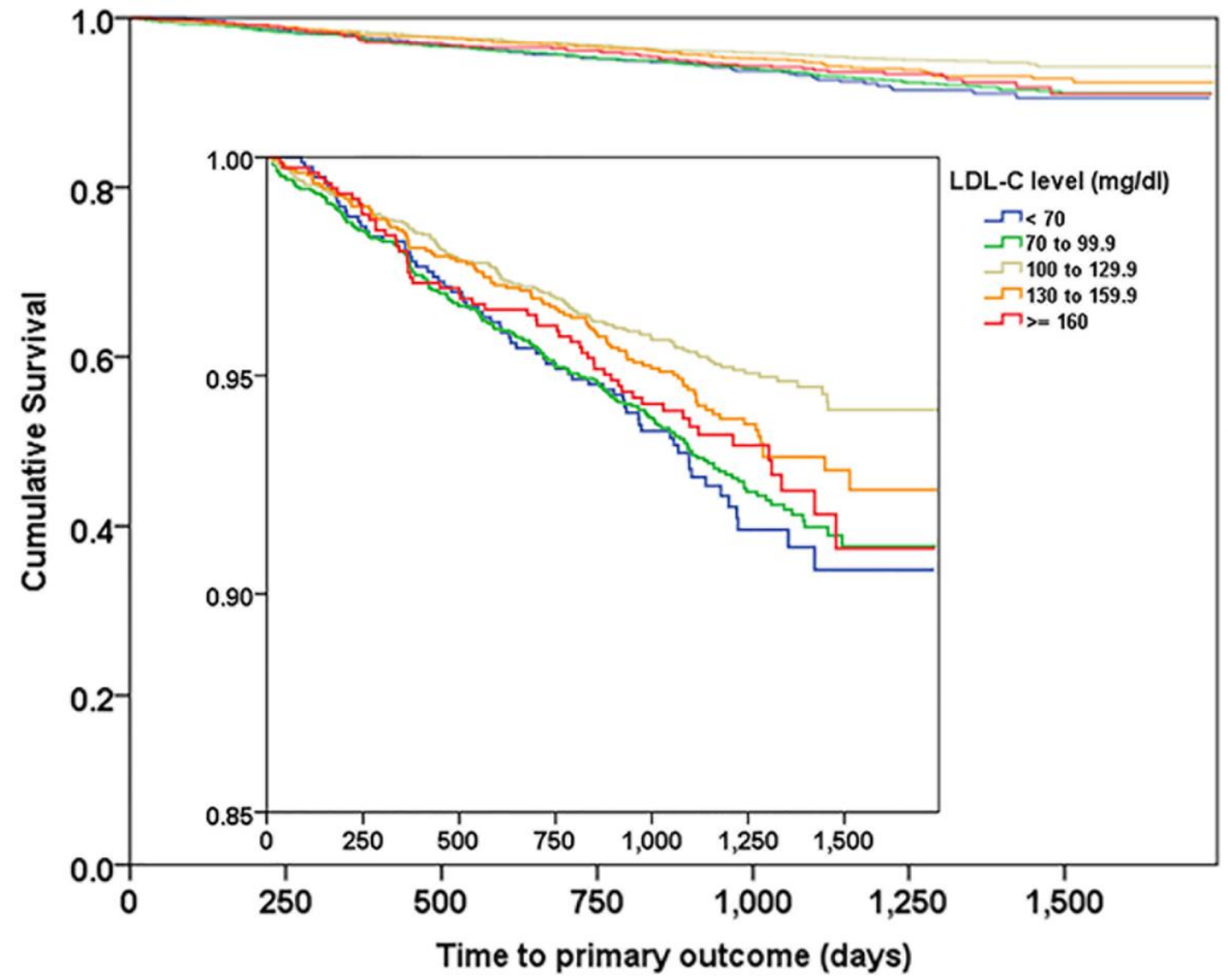

\begin{tabular}{|c|c|c|c|c|c|c|c|}
\hline & Baseline & Day 250 & Day 500 & Day 750 & Day 1000 & Day 1250 & Day 1500 \\
\hline$<70$ & 902 & 863 & 836 & 801 & 627 & 317 & 110 \\
\hline 70 to 99.9 & 2779 & 2687 & 2595 & 2512 & 2062 & 1125 & 361 \\
\hline 100 to 129.9 & 3057 & 2945 & 2865 & 2789 & 2344 & 1236 & 411 \\
\hline 130 to 159.9 & 1732 & 1665 & 1611 & 1558 & 1333 & 722 & 221 \\
\hline$>=160$ & 853 & 823 & 797 & 775 & 636 & 346 & 104 \\
\hline
\end{tabular}


Figure 2. Cumulative survival curves regarding primary outcome in the subgroup of patients in secondary prevention in the SPRINT cohort $(n=1562)$, depending on baseline LDL-C levels.

Multivariable Cox regression analyses showed a significant association between LDL$C$ and the primary event with an adjusted HR of 1.005 (1.002-1.009), $p=0.005$. ROC AUC did not reach significance with $0.54, p=0.087$. There were $7(0.4 \%)$ missing values for baseline LDL-C.

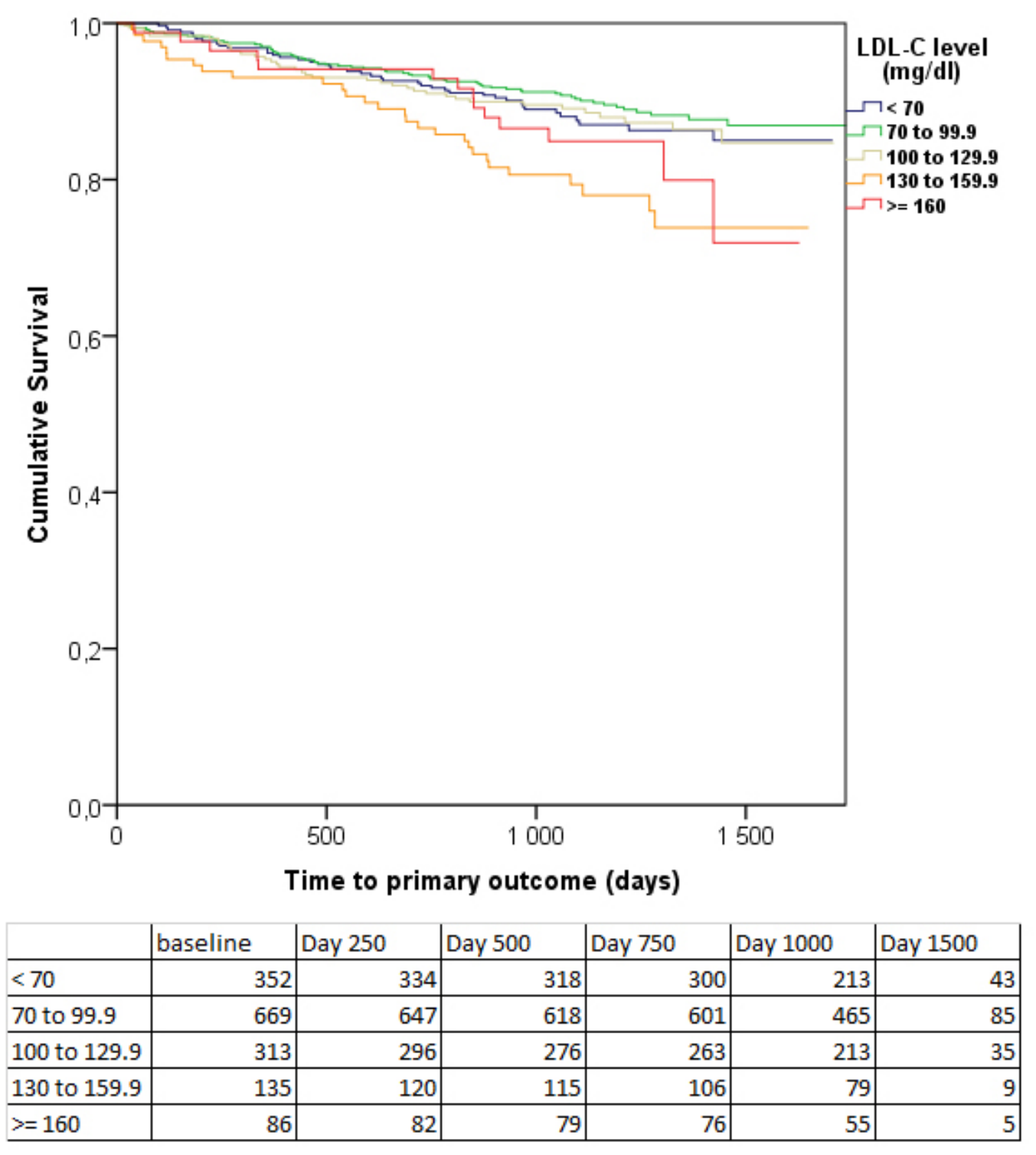

